\title{
Nonradial Pulsation among $\delta$ Scuti Stars
}

\author{
Michel Breger \\ Institute of Astronomy, University of Vienna, Austria
}

\begin{abstract}
The review examines recent observational and theoretical results on the nonradial pulsation of $\delta$ Scuti stars. The dominant pulsation modes are rotationally split $\mathrm{p}$ modes with $\ell=1$ and 2 , and radial orders 1 to 4 . Line-profile analyses also reveal the existence of additional high-degree modes. Nonradial pulsation is usually accompanied by slow amplitude and period variability. The small-amplitude single-mode variables are shown to be typical nonradial pulsators.

The interesting cases of 1 Mon and $\theta^{2}$ Tau are examined in more detail. The present status of asteroseismolgy of the $\delta$ Scuti stars is reviewed.

The periods of the four evolved $\delta$ Scuti variables studied are found to be decreasing, although stellar evolution predicts period increases due to larger radii. New work on the multiple periods of AI Vel shows that, apart from stellar evolution, an additional mechanism must exist to cause temporary period changes. It is argued that for a larger sample of stars the average period change must nevertheless reflect evolutionary changes.
\end{abstract}

\section{Introduction}

The $\delta$ Scuti stars are situated in the classical cepheid instability strip. A few are radial pulsators, while the majority pulsate with a large number of nonradial $\mathrm{p}$ modes simultaneously. They represent a transition between the cepheid-like large-amplitude radial pulsation of the classical instability strip and the ocean of nonradial pulsation occurring in the hot half of the H-R Diagram. Many excited modes show photometric amplitudes in excess of $0.001 \mathrm{mag}$, which makes it possible to study these stars photometrically. The position of $\delta$ Scuti stars on and slightly above the main sequence permits the asteroseismological comparison between oscillation data and stellar models in a region where the basic stellar structure is regarded as relatively well known.

The relationship between the $\delta$ Scuti stars and other pulsators in the hot part of the H-R Diagram is illustrated in Figure 1. We notice that, according to the recent studies, the $\delta$ Scuti stars are only one of many types of nonradial pulsators. The difference between the pulsation properties of these different types of stars must be a reflection of the different stellar structure and evolution.

Stars with dominant radial pulsation are found among Pop. II as well as slowly rotating Pop. I $\delta$ Scuti stars. The known radial pulsators usually have only one or 
two known periods as well as very large amplitudes with typical amplitudes of 0.5 mag. This is a factor 10 or 100 larger than the amplitudes of the typical nonradial pulsators.

The nonradial pulsations of $\delta$ Scuti stars found photometrically are low-order $(\ell \leq$ 3 ) and low-degree ( $\mathrm{n}=0$ to 4$) \mathrm{p}$ modes. A typical example is the star $4 \mathrm{CVn}$, for which the observed frequencies could be matched by rotationally split $p_{1}, p_{2}$ and $p_{3}$ modes with $\ell=2$ (Breger et al. 1990, Breger 1990a).

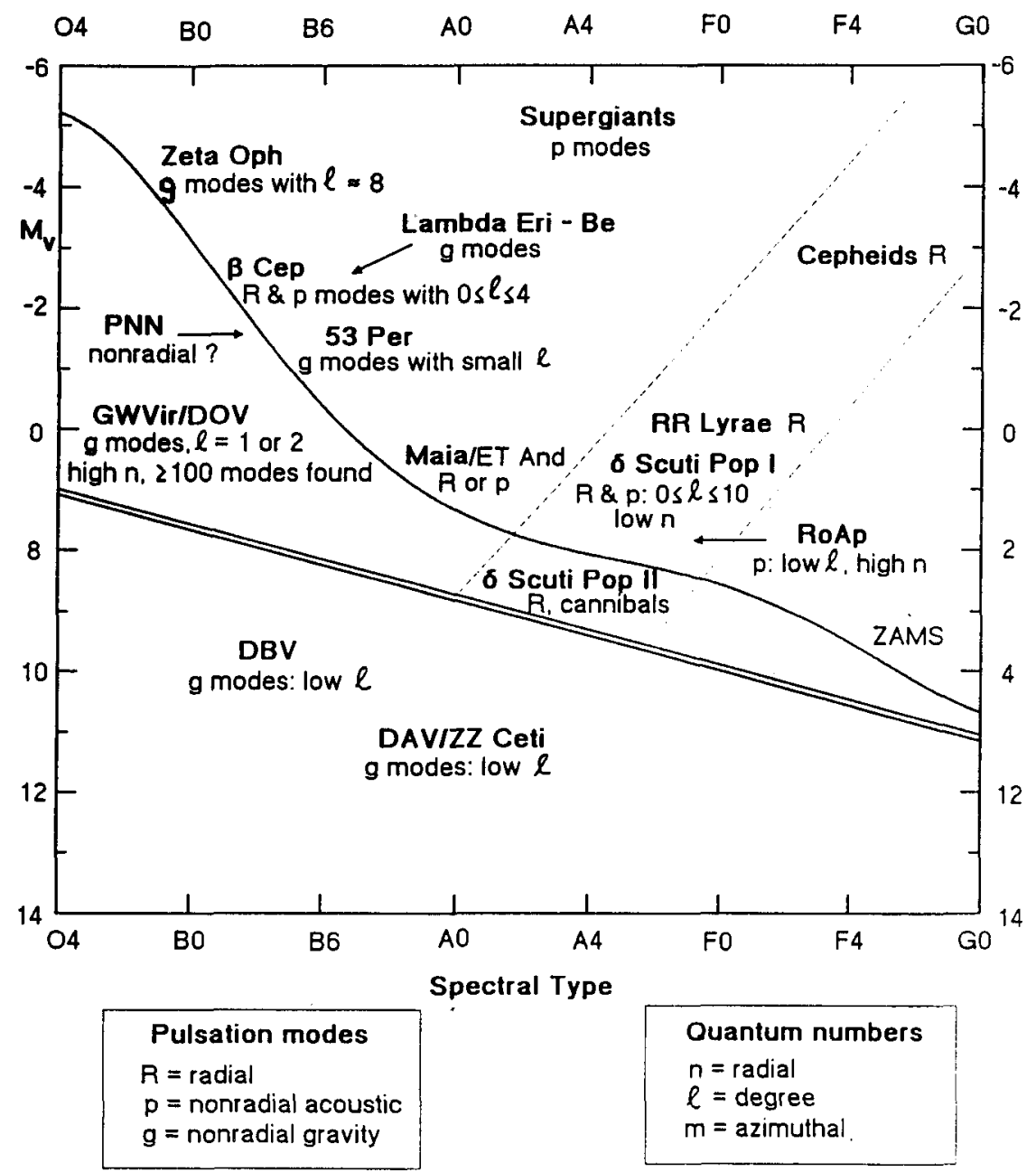

Figure 1 The position of the $\delta$ Scuti variables in relation to other types of pulsators in the hot part of the HRD. Some of the mode identifications and other recent information may be speculative. There is no universal agreement that the light variations of the $\lambda$ Eri stars are indeed caused by pulsation. 
While these nonradial low-order $\mathrm{p}$ modes are probably the most common modes found among $\delta$ Scuti stars, it must be noted that these modes are also the easiest to detect photometrically. On the other hand, the study of line-profile variations favors the detection of high-degree sectorial modes with $\ell=|\mathrm{m}|$. These high-degree modes are being studied by the group at the University of British Columbia (e. g. $|\mathrm{m}| \sim 10$ for $\gamma$ Bootis, Kennelly et al. 1992). For $\kappa^{2}$ Bootis, the available data can be matched by a low-degree mode $(\ell=0$ to 2 ) and a high-degree $\ell=|\mathrm{m}| \sim 12$ mode (Kennelly et al. 1991).

The multiperiodicity and the small amplitudes make the observational determination of the multiple periods quite difficult. In order to limit the effects of aliasing, photometric multisite campaigns around the globe have given the most promising answers. Only about a dozen nonradially pulsating $\delta$ Scuti stars can be regarded as being observed well enough for the multiperiod structure to be regarded as 'known'. Future work should probably concentrate on some very large campaigns utilizing telescopes on several continents in order to detect more of the large number of excited pulsation modes.

\section{Stars with only one or two periods}

A few $\delta$ Scuti stars appear to show only a single period of pulsation with a small amplitude of pulsation near 0.01 mag. Could these stars be radial pulsators such as the single or double mode stars with large amplitudes? Or are they nonradial pulsators such as the small-amplitude multiperiodic variables?

During the last few years three photometrically monoperiodic stars were examined in more detail in order to identify the pulsation mode.

The star $\tau$ Peg shows a single photometrically dominant frequency of 18.4052 cycles $\mathrm{d}^{-1}$ with an amplitude changing slowly from $0.005 \mathrm{mag}$ (or less) to $0.012 \mathrm{mag}$ over 13 years. The amplitude ratios and phase differences between observed light and color variations rule out radial pulsation. A nonradial $\mathrm{p}_{3}$ or $\mathrm{p}_{4}$ mode with $\ell=2$ fits the observed data.

The results obtained for $\tau$ Peg were dramatically confirmed by the simultaneous $u v b y$ photometry of 28 And and $\beta$ Cas (Rodriguez et al. 1992a, 1992b). In 28 And only the dominant 14.429 cycles $\mathrm{d}^{-1}$ frequency could be detected photometrically. Its amplitude varied from season to season, while the phase shifts and amplitude ratios showed that 28 And also is a nonradial pulsator. The mode could be identified as $\mathrm{p}_{3}$, $\ell=2$. Furthermore, for the star $\beta$ Cas the pulsation mode could be identified as a $\mathrm{p}_{2}$ or $\mathrm{p}_{3}$ mode with $\ell=1$.

We can conclude that the photometrically single-period pulsators with small amplitudes are not analogs of the large-amplitude variables such as AI Vel, which pulsate mainly with radial modes. These measurements of the three stars demonstrate that the low-amplitude single-mode pulsators resemble the nonradial multifrequency $\delta$ Scuti stars. Even the values of the radial overtone, $\mathrm{n} \sim 2$, and $\ell=1$ or 2 are typical for nonradially pulsating $\delta$ Scuti stars. 
Table 1: Observed and computed pulsation modes for 1 Mon

\begin{tabular}{ccl}
\hline Frequency & Value $\left(\right.$ cycles $\left.^{-1}\right)$ & Identification \\
\hline Observed & & \\
$\mathrm{f}_{1}$ & 7.217139 & $\ell=1, \mathrm{~m}=+1$, probably $\mathrm{p}_{1}$ \\
$\mathrm{f}_{2}$ & 7.346146 & Radial, probably $1 \mathrm{H}$ \\
$\mathrm{f}_{3}$ & 7.475268 & $\ell=1, \mathrm{~m}=-1$, probably $\mathrm{p}_{1}$ \\
Computed & & \\
$\mathrm{f}_{0}$ & 7.3555 & $\ell=1, \mathrm{~m}=0$ \\
\hline
\end{tabular}

\section{The nonradial pulsation modes of 1 Mon}

One of the simplest (?) observed examples of a nonradial $\delta$ Scuti pulsator is the star 1 Mon, which has been extensively studied and analysed by Balona and Stobie (1980). They could represent the observed variations of 1 Mon by a set of three almost equidistantly spaced frequencies and various harmonics and combinations of these frequencies. The relatively large amplitudes together with the excellent data and analyses suggest that the frequency determinations should be reliable. Furthermore, the mode identifications by Balona and Stobie on the basis of color phase and amplitude relations were confirmed through line-profile analyses by Smith (1982).

It can be seen in Table 1 that the three frequencies found by Balona and Stobie almost form an almost exact frequency split with a deviation of only $\Delta f=\left(f_{3}-f_{2}\right)$ $-\left(f_{2}-f_{1}\right)=0.000115$ cycles $d^{-1}$. This value corresponds to the inertial (observer's) frame.

The fact that an almost exact frequency split is observed can hardly be accidental. Due to the second-oder effects, for rotating stars rotational mode splitting does not lead to equidistantly spaced frequencies. On the basis of the identification of $f_{1}$ and $f_{3}$ with $\ell=1$, we can calculate the expected value of the central $m=0$ frequency through the following equation

$$
\sigma_{\mathrm{m}}=\sigma_{0}+\left(\mathrm{C}_{\mathrm{L}}-1\right) \mathrm{m} \Omega+\mathrm{D}_{\mathrm{L}} \mathrm{m}^{2} \Omega^{2} / \sigma_{0}
$$

where $\sigma$ represents the frequency of pulsation of mode with azimuthal quantum number $\mathrm{m}, \Omega$ is the rotational frequency, while $\mathrm{C}_{\mathrm{L}}, \mathrm{D}_{\mathrm{L}}$ are coefficients depending on the model and pulsation mode. For the present calculation we determine the rotational frequency, $\Omega$, from the observed frequency separation of the $m=+1$ and $m=-1$ modes for a $p_{1}$ mode and find $\Omega=0.131$ revolutions per day. This value is reasonable and with a radius of 2.2 solar radii (given by Balona and Stobie) predicts the rotational velocity of $14.5 \mathrm{~km} \mathrm{~s}^{-1}$ (observed $v \operatorname{sini} \sim 15 \mathrm{~km} \mathrm{~s}^{-1}$ ). 
Three models were chosen for the $\mathrm{C}_{L}$ and $\mathrm{D}_{L}$ coefficients: the polytrope model by Saio (1981), the ZAMS model as well as the evolved model, both computed by Dziembowski and Goode (1992). It was found that for the $\mathrm{p}_{1}, \ell=1$ modes all three models give similar answers, so that only the results for the ZAMS model are shown in Table 1.

The calculations show that even the low rotational velocity of $\sim 15 \mathrm{~km} / \mathrm{s}$ leads to nonequal frequency splitting. In fact, the observed spacing is more than a factor of 100 times more equidistant than the predictions for the three $\ell=1$ modes! If the central mode is indeed radial (and not the central $\ell=1$ mode), then the observed equidistant splitting may not be a problem. Since in the stellar co-rotating frame the three frequencies have essentially the same value, the nonradial modes might be in resonance with the radial mode.

The picture presented above is internally consistent, but leaves two questions:

(i) Why do the frequency values of the $\ell=0$ (radial) and the two $\ell=1$ modes agree to better than one part in $10^{4}$ in the co-rotating frame?

(ii) Why are the central $\ell=1, \mathrm{~m}=0$ mode and its beating with the radial mode (beat period of about 100 days) not seen photometrically?

\section{Multiple nonradial pulsation modes and asteroseismology}

The resolution of the discrepancies between observations and theory concerning the radial period ratio, $\mathrm{P}_{0} / \mathrm{P}_{1}$ (Petersen 1992) can be regarded as an asteroseismological success. The observed value of 0.773 for Pop. I $\delta$ Scuti stars can now be matched by the new opacity tables with higher opacity values.

Among the nonradial oscillators, the simultaneous excitation of a large number of pulsation modes makes most $\delta$ Scuti stars very difficult to study observationally. On the other hand, the amount of astrophysical information that can be derived from the study of these pulsation modes depends directly on the number of modes that can be successfully identified in a star. The procedure can be divided into three stages:

(i) the observational determination of the excited multiple frequencies. This usually requires lengthy multisite photometric campaigns in order to decrease the 1 cycle $\mathrm{d}^{-1}$ aliasing problem,

(ii) the identification of the multiple frequencies with specific pulsation modes. The methods involve pattern recognition in frequency space, consideration of $Q$ values (see Breger 1989), amplitude and phase information between light and color variations (Balona and Stobie 1979, Watson 1988) as well as matching line-profile variations with models (Smith 1982),

(iii) the comparison of the frequencies with those predicted by a variety of models in order to obtain additional information on stars.

Dziembowski and Goode (1992) point out that patterns seen in the frequency spectrum could be misidentified since different orders can overlap and since we only see the amplitudes above a certain observational limit. For evolved stars, this difficulty is amplified by the possible development of $\mathrm{g}$-mode type behavior in the interior for low-order nonradial p modes (see Dziembowski and Krolikowska 1990, Dziembowski 
and Goode 1992). On the other hand, the trapped modes can provide an asteroseismological test for convective overshooting theories (Dziembowski and Pamyatnykh 1991).

The challenge could be met by two improvements to the present technique:

(i) for each star examined observationally specific stellar models should be computed,

(ii) more (true) modes need to be identified for each star studied observationally. For many $\delta$ Scuti stars only the modes with larger amplitudes can be extracted from. the photometric data due to the presence of noise and observational errors. Better statistical methods to extract multiple periods will probably only lead to a small improvement. The major improvement will probably be achieved through a further increase in the quantity and quality of the photometric data. In practice this might mean that more attention should be given to actually achieving the \pm 1 millimag precision presently possible with standard photoelectric photometers.

The second point concerning additional frequencies can be illustrated with an example. The star $\theta^{2}$ Tau was examined in two large multisite campaigns (Breger et al. 1987, 1989). The five frequencies found in the first campaign were confirmed by the second campaign, which also showed the constancy of the amplitudes. However, the data spanning six years contains more frequencies of pulsation. Their small amplitudes of pulsation less than 0.001 mag makes the identification of specific peaks in the power spectrum somewhat speculative; for this reason their values were not published.

Figure 2 shows the power spectrum of $\theta^{2}$ Tau after prewhitening of the five published frequencies in the 13.23 to 14.61 cycles $\mathrm{d}^{-1}$ range. The remaining power is concentrated in two regions. The high-frequency power occurs around the value of twice the frequencies already identified. We can speculate that, if real, this corresponds to the sum of two coupled frequencies. The lower frequency band is in the frequency range of the five frequencies which had already been identified and prewhitened. This means that more frequencies are probably excited. If one accepts the mode identifications derived from color and amplitude data $(\ell=0,1$ and 2$)$, the

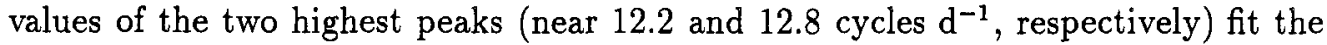
expected pattern! However, until more observations are available, these arguments must regarded as speculative.

Apart from illustrating the improvements suggested above, the example demonstrates that the star $\theta^{2}$ Tau shows considerable asteroseismological potential. A model for a star similar to $\theta^{2}$ Tau has been calculated by Dziembowski and Goode. If the new frequencies are indeed confirmed, then this star might provide the opportunity to compare the rotational splitting of $\ell=1$ and $\ell=2$ together with a 'normalization' of the frequencies relative to the radial mode at $13.23 \mathrm{c} / \mathrm{d}$.

We expect that future observations and models will lead to direct comparisons of the rotational mode splitting coefficients and permit the probing of the depth dependence of $\Omega$. Furthermore, the study of pulsational amplitudes and their selection rules provides additional means to test stellar models. 

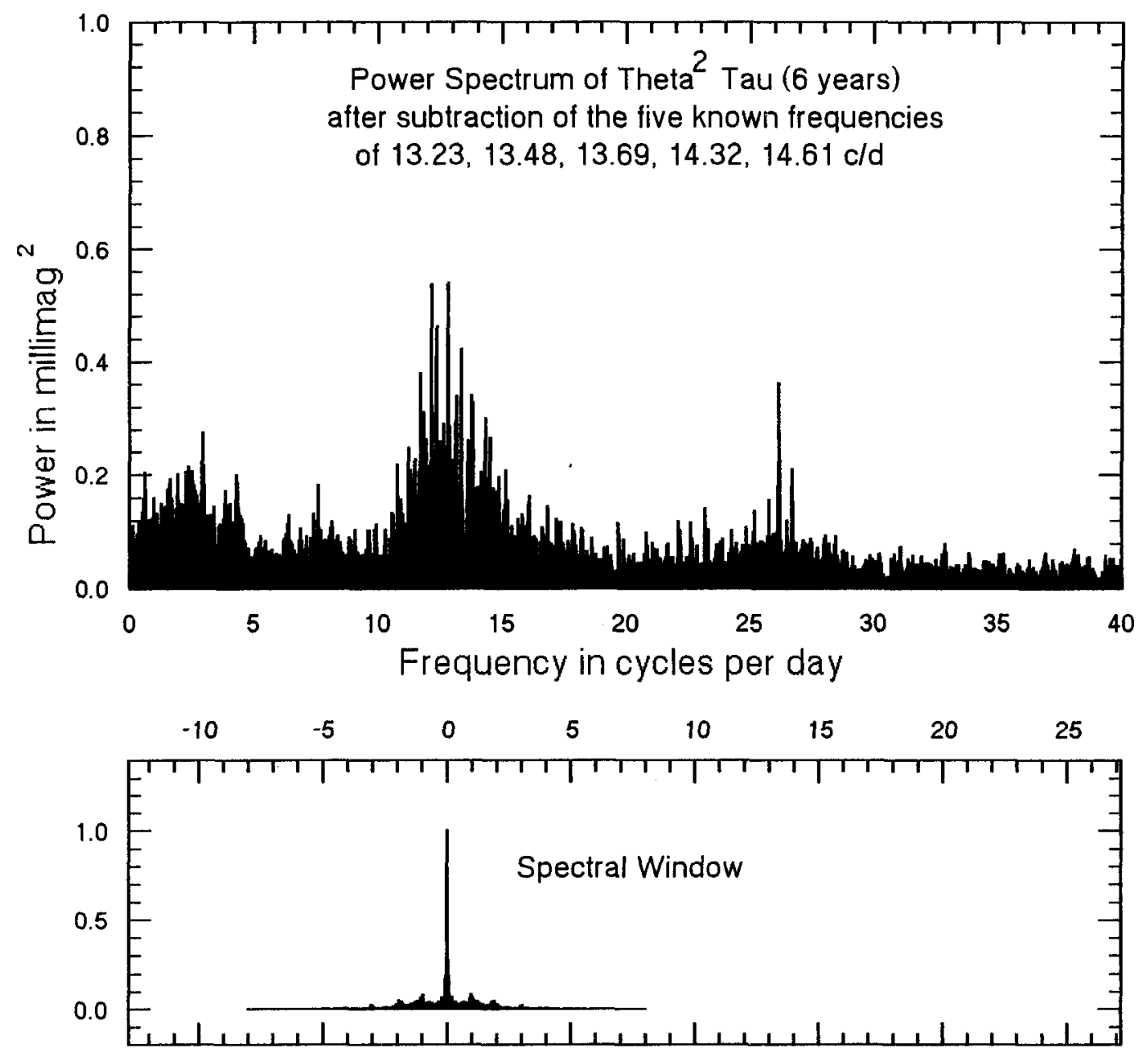

Figure 2 Power spectrum of $\theta^{2}$ Tau after prewhitening of the five published frequencies. The diagram shows that several additional frequencies with small amplitudes are also excited in the star.

\section{Period changes and evolution}

The period changes caused by the stellar evolution in and across the Lower Instability Strip permit an observational test of stellar evolution theory, provided that other physical reasons for period changes can be excluded. The period-density relation predicts

$$
(1 / \mathrm{P}) \mathrm{dP} / \mathrm{dt}=-0.69 \mathrm{dM}_{\mathrm{bol}} / \mathrm{dt}-\left(3 / \mathrm{T}_{\text {eff }}\right) \mathrm{T}_{\text {eff }} / \mathrm{dt}+(1 / \mathrm{Q}) \mathrm{dQ} / \mathrm{dt}
$$

The predicted values of $(1 / \mathrm{P}) \mathrm{dP} / \mathrm{dt}$ from $10^{-10}$ to $10^{-7}$ per year should be observable. 
At the last meeting of this pulsation series in Bologna, the observed period changes were compared with values calculated from theoretical evolutionary tracks (Breger $1990 \mathrm{~b})$. For the four evolved $\delta$ Scuti stars the observed period decreases were in contradiction to the radius increases predicted from stellar evolution. Could there be other physical explanations for the observed period decreases?

Guzik and Cox (1991) have examined possible explanations for the period decreases seen in evolved $\delta$ Scuti stars. While their models indeed predicted smaller period increases, period decreases were not found.

The recent results for the star AI Vel (Walraven, Walraven and Balona 1992) may indicate a solution to the dilemna. They have found that only one of the two radial frequencies shows a period increase, while the second frequency was essentially constant. The example of AI Vel shows that the (slow) stellar evolution is not the only mechanism to generate changes in the periods of pulsation. This means that for an individual star the conversion of observed period changes into stellar evolution rates (e.g. radius changes) has to be applied with caution. On the other hand, the nonevolutionary period changes should cancel out for a larger group of stars. This can be seen from the fact that the $\delta$ Scuti stars on the whole obey a period-luminositycolor relation.

The fact that all four evolved $\delta$ Scuti stars show period decreases may conceivably be a reflection of an additional period changing mechanism (leading to random changes) coupled with small-number statistics. A doubling of the number to eight stars should provide the required statistical significance.

Part of this investigation has been supported by the Austrian Fonds zur Förderung der wissenschaftlichen Forschung.

\section{References:}

Balona, L. A. and Stobie, R. S. 1979, MNRAS, 189, 649

Balona, L. A. and Stobie, R. S. 1980, MNRAS, 190, 931

Breger, M. 1989, Comm. Asteroseismology, 7, 1, Vienna: Austrian Acad. Sciences

Breger, M. 1990a, A\&A, 240, 308

Breger, M. 1990b, ASP Conf. Ser., 11, 263

Breger, M., Garrido, R., Huang, L., Jiang, S.-Y., Guo, Z.-h., Frueh, M., Paparo, M. 1989, A\&A, 214, 209

Breger, M., Huang, L., Jiang, S.-y., Guo, Z.-h., Antonello, E., Mantegazza, L. 1987, A\&A, 175,117

Breger, M., McNamara, B. J., Kerschbaum, F., Huang, L., Jiang, S.-y., Guo, Z.-h., Poretti, E. $1990, A \& A$ 231, 56

Dziembowski, W. A., and Goode, P. R. 1992, ApJ, in press

Dziembowski, W. A. and Krolikowska, M. 1990, Acta Astron. 40, 19

Dziembowski, W. A. and Pamyatnykh, A. A. 1991, A\&A, 248, L11

Fitch, W. S. 1981, ApJ, 249, 218

Guzik, J. A. and Cox, A. N. 1991, in Delta Scuti Newsletter, 3, 6, Vienna: Austrian Acad. Sciences

Kennelly, E. J., Walker, G. A. H. and Hubeny, I. 1991, PASP, 103, 1250 
Kennelly, E. J., Yang, S., Walker, G. A. H. and Hubeny, I. 1992, PASP, 104, 15

Petersen, J. O. 1992, A\&A, in press

Rodriguez, E., Rolland, A., Lopez de Coca, P., Garrido, R. and Gonzalez-Bedolla, S. F. 1992a, A\&AS, in press

Rodriguez, E., Rolland, A., Lopez de Coca, P., Garrido, R. and Mendoza, E. E. 1992b, $A \& A$, in press

Saio, H. 1981, ApJ, 244, 299

Smith, M. A. 1982, ApJ, 254, 242

Walraven, Th., Walraven, J. and Balona, L. A. 1992, MNRAS, 254, 59

Watson, R. D. 1988, A\&SS, 140, 255

\section{DISCUSSION}

BALONA: The reason why we do not see a beating between the $\ell=0$ and the $\ell=1$, $m=0$ modes in 1 Mon may be due to the inclination of the axis of rotation, $i$. For $i \sim 90 \mathrm{deg}$, the observed amplitude of $\ell=1, m=0$ is close to zero whereas $\ell=1$, $m= \pm 1$ is close to the maximum value.

BREGER: Such an inclination would explain the observations. It is regrettable that the small value of $v \operatorname{sini} \sim 15 \mathrm{~km} \mathrm{~s}^{-1}$ makes it difficult to derive the inclination by comparing this value with the predicted value of the rotational velocity, $v$.

MATTHEWS: What limit does the frequency spacing of 1 Mon set upon the secondorder rotational splitting term $D_{\mathrm{L}}$ and what could this mean for the star's structure? BREGER: If the central frequency of the observed triplet were the missing $\ell=1$, $\mathrm{m}=0$ mode, no model I have seen can predict such a small $\mathrm{D}_{\mathrm{L}}$ value. After all, waves travelling in the same direction as stellar rotation have a weaker effective gravity (and therefore a different frequency) than waves travelling in the opposite direction. 